\title{
Efectividad de seis juegos de mesa \\ en enseñanza de conceptos básicos de nutrición
}

\author{
Effectiveness of six table games \\ in teaching basic \\ nutrition concepts
}

\begin{abstract}
Objective: To evaluate the effectiveness of six board games to teach food and nutrition, personal hygiene and physical activity in children of elementary school education. Methods: Cluster randomized controlled study of children in basic education (7-12 year old) enrolled in the city schools from the District of Bogotá. Two local schools were selected and randomized to intervention or control group. In the intervention group were 472 children and 487 were in the control group. Children's knowledge was assessed using a questionnaire at three different times. To analyze the model we used generalized estimating equations (GEE) for repeated measures to study the effect of the intervention over time. Results: In relation to knowledge the intervention group increased its score in the second time, on a scale of 0-100 steps from 49.4 to $61.8(p<0.001)$ and remained at the time. In the control group there was not a change in the score of time. Conclusions: The results suggest that the six games can be effective tools in teaching basic concepts in food, nutrition and health to schoolchildren.
\end{abstract}

Key words: Effectiveness, board games, concept, nutrition education, nutrition.

\section{INTRODUCCIÓN}

Son muchas y muy variadas las estrategias metodológicas de utilidad en el proceso de enseñanza-aprendizaje para el logro de los objetivos. La elección del tipo de estrategia a utilizar en cada situación dependerá de diversos factores, entre los que destacan las características de la población objetivo, los recursos humanos, materiales y la disponibilidad y acceso a los distintos medios.

En la década de los noventa se empezó a incorporar nuevos modelos educativos rompiendo con el enfoque tradicional de la educación en nutrición, utilizando los juegos como estrategias de enseñanza aprendizaje para promover estilos de vida saludables y para a prevención y control de enfermedades. El juego como estrategia pedagógica ha sido estudiado por muchos autores, pero fue Froebel el primero en incorporarlo a la pedagogía, reconociendo su valor educativo. Según dicho autor el juego es el medio más adecuado para introducir al niño al mundo de la cultura, la creatividad, la sociedad y el servicio a los demás, por lo que debe considerarse como parte esencial en la educación, por ser para el niño una
Martha Constanza Liévano $F$. Martha Helena Leclercq B.

Departamento de Nutrición y Bioquímica, Facultad de Ciencias, Pontificia Universidad Javeriana, Bogotá, Colombia.

Dirigir la correspondencia a:

Nutr.

Martha Constanza Liévano Fiesco

Carrera. 7 No. 43-82

Oficina 506 Edificio 52 Bogotá, Colombia

Telefax: 3208320 Ext. 4059

E-mail:mlievano@javeriana.edu.co

Este trabajo fue recibido el 16 de Octubre de 2012 y aceptado para ser publicado el 5 de Mayo de 2013.

función natural $(1,2)$.

Millians (1999) (3) sugiere que los juegos educativos y en particular los juegos de mesa son apropiados para lograr un nivel cognitivo de estudiantes de educación básica y media, proporcionando un medio para explorar y a la vez divertido, facilitando el aprendizaje (4), por lo que pueden ser una muy buena estrategia para el desarrollo de programas de educación en salud (5).

Existen en la literatura reportes de investigaciones en la que han evaluado diferentes estrategias lúdicas para la promoción de hábitos saludables de nutrición y de actividad física en niños, que han puesto de manifiesto sus posibilidades cognitivas $(2,6,7)$. En Colombia, son pocos los reportes de experiencias al respecto, pero el grupo de investigación en educación nutricional del Departamento de Nutrición y Bioquímica de la Pontificia Universidad Javeriana ha diseñado, como método de enseñanza-aprendizaje en el ámbito escolar, seis juegos secuenciales para la promoción de estilos de vida saludable (estilos de vida saludable, higiene personal y de los alimentos y actividad física). Los juegos diseñados son: 
Concéntrese: "Busca la pareja y ganarás"; Lotería: "Nutrilotto"; Cartas: "Nutri-cartas"; Bingo: "Nutri-salud"; Dominó: "Nutri-derechos" y Escalera: "Lleguemos al mundo mágico de la alimentación".

Sin embargo, los juegos desarrollados localmente no habían sido sometidos a un proceso de evaluación, actividad indispensable para determinar si logran los objetivos para los cuales fueron propuestos.

La presente investigación tuvo por objetivo determinar la efectividad de los juegos de mesa desarrollados como estrategia de enseñanza - aprendizaje para la adquisición de conocimientos básicos en alimentación y nutrición, higiene personal y actividad física en niños escolares. Aun cuando está demostrado que para prevenir la malnutrición es necesario lograr cambios conductuales en los aspectos de dieta y actividad física, el primer paso necesariamente debe ser la adquisición de un nivel básico de conocimientos en estos aspectos (8).

\section{SUJETOS Y MÉTODOS}

Se realizó un estudio experimental controlado con aleatorización por conglomerados, que ha sido reconocido como el diseño más adecuado para evaluar el impacto de programas educativos (9).

Para el presente estudio se decidió tomar como unidad de aleatorización los salones de clase (conglomerados) de los colegios seleccionados.

En base al listado de colegios de educación básica primaria (niños 7 -12 años) se seleccionaron aleatoriamente dos, la asignación del colegio incluyendo tanto jornada de la mañana como de la tarde a cada uno de los grupos (intervención y control) se realizó también de manera aleatoria, una vez se contó con el aval de las directivas de las instituciones para participar en el proyecto.

Utilizando el programa Sampsize Vr. 6.06 (10) se calculó el tamaño de la muestra requerida corrigiendo por el factor de inflación o efecto del diseño (11-13). Con el fin de corregir las posibles pérdidas que se pudieran generar durante el estudio, el tamaño de la muestra se aumento un $9.1 \%$ quedando el número de conglomerados en 24 y el tamaño total de la muestra de 908 es decir, aproximadamente 454 niños por cada grupo.

La selección de los conglomerados se hizo mediante muestreo estratificado, con el fin de asegurar representación de todos los niveles de primero a quinto grado de educación básica de los colegios.

El proyecto contó con el aval del comité de investigación y Bioética de la Facultad de Ciencias de la Pontificia Universidad Javeriana. Para cada niño se empleó un formato individual de asentimiento y de consentimiento informado, el cual fue elaborado teniendo en cuenta los lineamientos sugeridos por Lozano y Ruiz (14), el cual incluyó información sobre el estudio, así como las responsabilidades y compromisos tanto del investigador como de los participantes.

Los niños tanto del grupo de intervención como del control fueron evaluados pre y post intervención en función del puntaje obtenido en una escala de 0 (puntaje mínimo) a 100 (puntaje máximo), con base a una lista de preguntas relacionadas con alimentación y nutrición, derechos de los niños, higiene personal y de los alimentos y actividad física. Se empleó un cuestionario auto-administrado desarrollado por las autoras de los juegos.

En condiciones basales se aplicó un cuestionario autoadministrado tanto en el grupo de intervención como en el control.

La fase de intervención tuvo una duración aproximada de dos meses, durante los cuales, cada semana y con cada uno de los conglomerados del grupo de intervención, se jugó cada uno de los seis juegos, mientras que los niños del grupo control no fueron intervenidos. Los juegos en los que participaron los niños fueron:

- Concéntrese "Busca la pareja y ganaras"

Tema: Origen de los alimentos

Objetivo: Al término del juego concéntrese "busca la pareja y ganarás", los niños estarán con capacidad de identificar a que reino de la naturaleza pertenecen los alimentos que hacen parte de la alimentación diaria.

- Cartas "Nutri-cartas".

Tema: Funciones de los alimentos.

Objetivo: Al término del juego de cartas "Nutri-cartas" los niños estarán con capacidad de identificar la función que cumplen los alimentos pertenecientes a cada uno de los tres reinos para un adecuado crecimiento y desarrollo.

- Rompecabezas "Armando el tren de la alimentación saludable"

Tema: Conozcamos nuestros amigos los alimentos.

Objetivo: Al terminar el rompecabezas "Armando el Tren de la Alimentación Saludable" los niños estarán con capacidad de identificar los alimentos que se encuentran en cada vagón del tren, así como su función y la importancia de incluirlos diariamente en su alimentación para un adecuado crecimiento y desarrollo.

- Bingo "Nutri-salud".

Temas: higiene personal y de los alimentos y actividad física.

Objetivo: Al término del bingo "Nutrisalud" los niños estarán en capacidad de:

Identificar las normas de higiene personal y de los alimentos como parte de su rutina diaria para prevenir enfermedades.

Enumerar las ventajas de practicar actividad física diariamente para mantener un estado de salud adecuado.

- Lotería "Nutriderechos"

Tema: Los derechos del niño.

Objetivo: Al término del juego con la lotería "NutriDerechos" los niños estarán con capacidad de Identificar sus derechos como niños.

- Escalera "Lleguemos al mundo mágico de la alimentación".

Objetivo: Al término del juego de escalera "Lleguemos a Nutrilandia" los niños estarán en capacidad de:

- Identificar los alimentos y su origen.

- Identificar los alimentos que deben incluir diariamente en su alimentación para un adecuado crecimiento y desarrollo.

-Enunciar las reglas de higiene personal y de los alimentos como parte de su rutina diaria para prevenir enfermedades.

- Enunciar las ventajas de la actividad física diaria para mantener un estado de salud adecuado.

- dentificar sus derechos como niños.

La evaluación del desenlace se realizó en dos momentos: el primero una semana después de finalizar las actividades educativas con los seis juegos en cada uno de los conglomerados y la segunda tres meses después de ésta evaluación (es decir, tres meses y una semana después de finalizar las actividades educativas). En cada una de estas evaluaciones se aplicó nuevamente el cuestionario estandarizado tanto en los grupos de intervención como en los controles.

La información recolectada se tabuló utilizando el pro- 
grama Epi Info Versión 3.3.2 y se analizó con Stata 10.0®. Un primer análisis consistió en comparar los grupos de tratamiento en sus condiciones basales y en sus características demográficas (edad y género). Posteriormente, se realizó un análisis exploratorio de los datos, para describir la distribución de las variables y la posibilidad de aplicar métodos basados en la distribución normal. Se utilizaron las pruebas $\mathrm{t}$ de Student para muestras independientes y la prueba $U$ de Mann Whitney para comparar los datos entre el grupo de intervención y el control.

Por último, se utilizó un modelo de ecuaciones de estimación generalizada (GEE); para estudiar el efecto de la intervención en los puntajes a través del tiempo15-17. Se evaluó la existencia de interacciones, introduciendo las variables independientes multiplicativas con pruebas de significación estadística y se mantuvieron en el modelo las interacciones que fueron estadísticamente significativas.

\section{RESULTADOS}

Se seleccionaron 13 conglomerados en cada uno de los colegios, en el grupo de intervención 7 salones de la mañana ( $n=257$ niños) y 6 de la tarde $(n=215)$, y en el grupo control 6 por la mañana $(n=224)$ y 7 por la tarde $(n=263)$ (figura 1).

En condiciones basales no hubo diferencias entre el grupo experimental y el control en cuanto a la edad (promedio $8.91 \pm 1.62$ en grupo de intervención y $9.10 \pm 1.48$

\section{FIGURA 1}

Diagrama de flujo de selección, asignación y participación en el estudio.

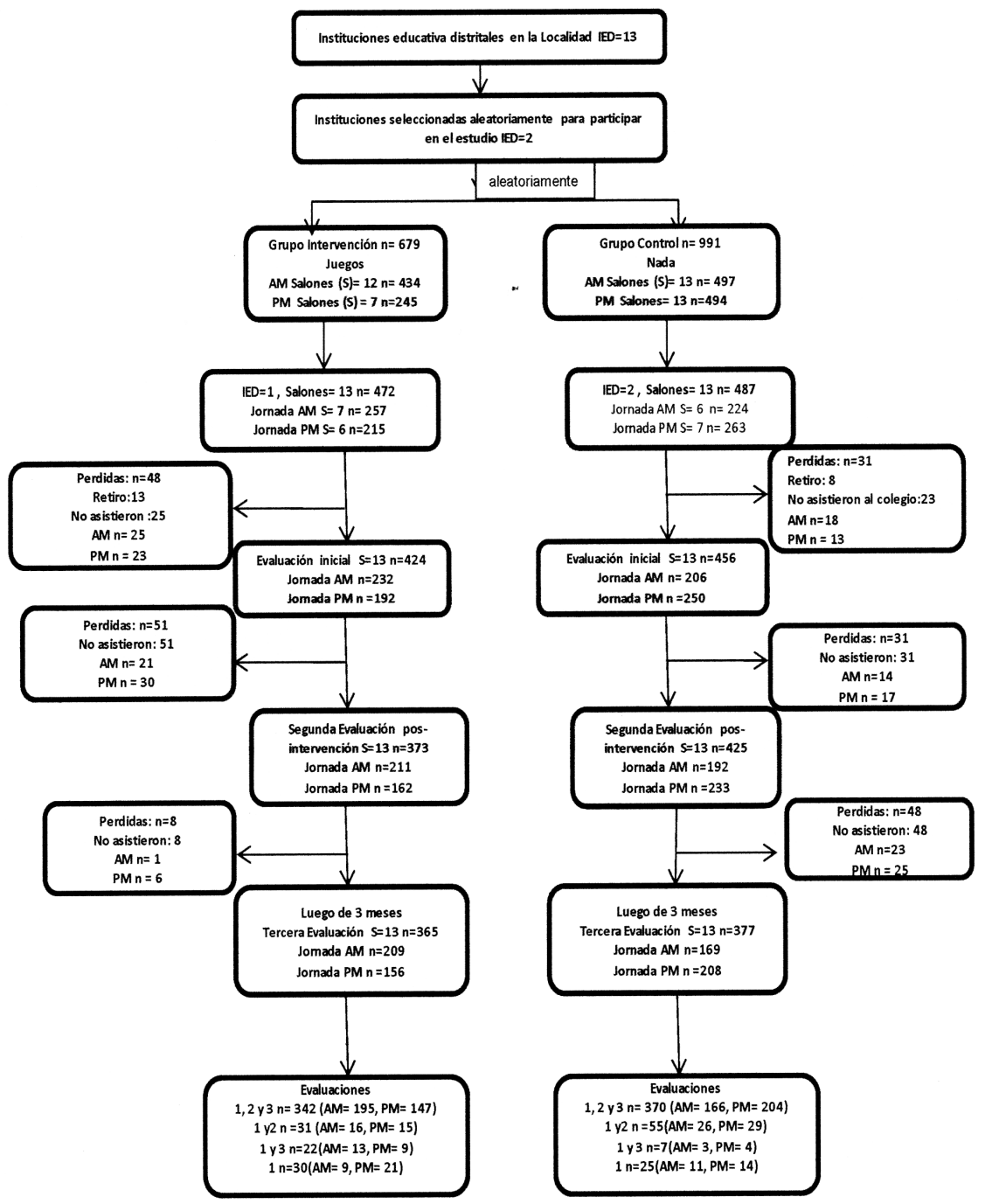


en el control) $(p=0.073)$, la distribución por sexo (54\% sexo masculino y $46 \%$ femenino en el grupo de intervención y en el grupo control $52.6 \%$ de sexo masculino y $47.4 \%$ de sexo femenino) $(p=0.68)$.

\section{Evaluación de los conocimientos}

En la figura 2 se presentan las calificaciones obtenidas por cada uno de los grupos en los tres momentos (momento 1: antes de la intervención, momento 2: una semana después de que el grupo experimental recibiera el último juego, y momento 3: tres meses después de la segunda evaluación). Se observa que el grupo control presenta mejores calificaciones en el momento 1 las cuales disminuyen un poco en el segundo momento y se mantienen a través del tiempo. Por otro lado, el grupo intervención incrementa su puntaje en el segundo momento y se mantiene tres meses después.

Para estudiar el efecto de la intervención educativa en los conocimientos a través del tiempo, se calculó la diferencia entre el puntaje obtenido por cada niño en cada uno de los tres momentos. La tabla 1 muestra estas diferencias. En el grupo control se observó una diferencia reducida entre el momento 2 y el momento 1, y en los otros momentos no se presentaron diferencias. En el grupo de intervención hubo diferencias en los puntajes en el momento 2 en comparación con el momento 1, y en el momento 3 con el momento 1, pero hubo muy poca diferencia entre el momento 2 y el momento 3. Al comparar estas diferencias entre grupos (intervención y control) se observó que existen diferencias estadísticamente significativas, entre la nota 2 y la nota 1 y entre la nota 3 y la nota $1(p<0.001)$.

Al hacer la evaluación por medio del modelo (GEE), usando como variable dependiente las calificaciones obtenidas por los grupos en los diferentes momentos y ajustando por las covariables, las estimaciones de los parámetros co-

\section{FIGURA 2}

Puntaje obtenido por los dos grupos en los diferentes momentos.

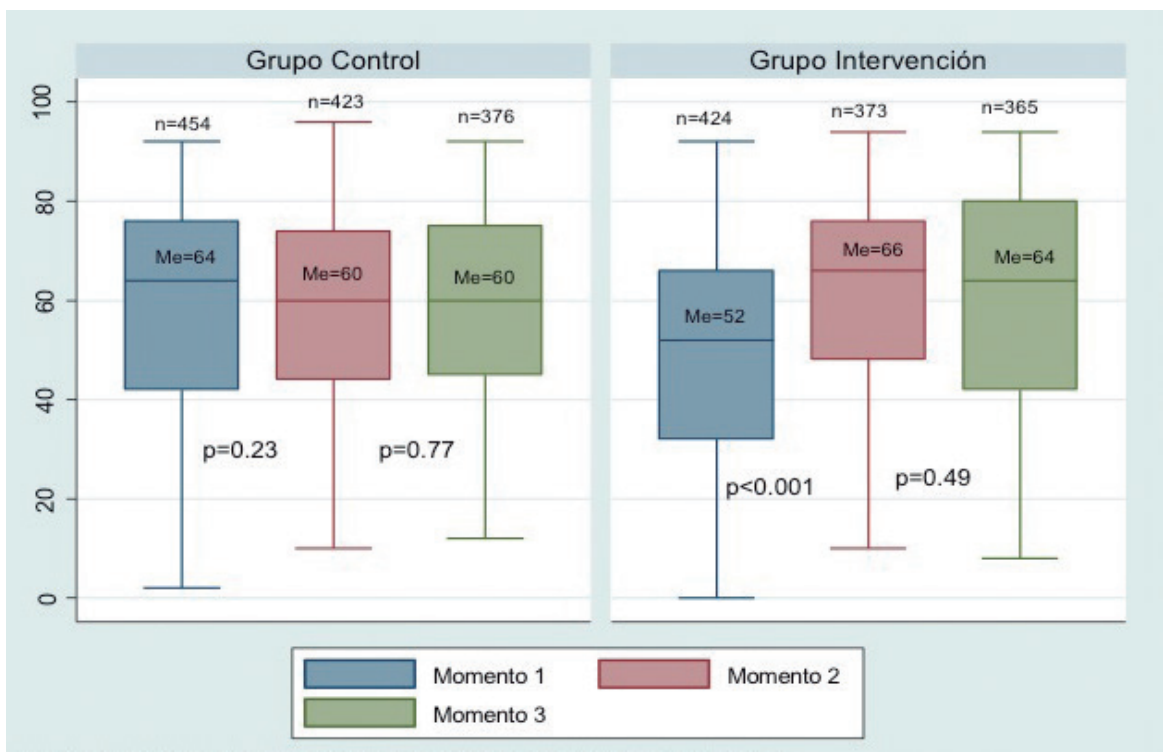

TABLA 1

Diferencias entre los puntajes obtenidos por los dos grupos en los diferentes momentos.

Diferencias entre las calificaciones

Nota 2 - Nota 1

Nota $3-$ Nota 2

Nota 3 - Nota 1

\section{Grupo intervención}

n Mediana (P25;P75)

373

342

365
$10(-7 ; 30)$
$2(-20 ; 18)$
$10(-4: 24)$
Grupo control

Mediana (P25;P75)

$$
\begin{gathered}
4(-12 ; 16) \\
0.00(-4 ; 4) \\
0.00(-.14 ; 14)
\end{gathered}
$$

$\mathrm{p}$

$<0.001^{*}$

$<0.001^{*}$

$<0.001^{*}$

Diferencia estadísticamente significativa. Prueba de U Mann-Whitney. 
rrespondientes a los efectos de interacción entre momentointervención resultaron estadísticamente significativas, por lo cual el efecto de la intervención fue diferente en los tres momentos (tabla 2).

\section{DISCUSIÓN}

De acuerdo con la búsqueda, existen pocos reportes en la literatura tanto a nivel nacional como internacional que hayan utilizado juegos de mesa como estrategia metodológica en la promoción de estilos de vida saludables en población escolar.

En este este estudio se utilizaron seis juegos de mesa tradicionales diseñados como parte de un programa de educación alimentaria y nutricional para la enseñanza de diversos conceptos de alimentación y nutrición, higiene personal y práctica de actividad física en niños de primero a quinto grado de educación básica.

Aunque el nivel de conocimientos basales era bajo en ambos grupos (49.4 puntos sobre 100 en el grupo intervención y 57.1 puntos en el grupo control) se logró incrementar los conocimientos en los niños que participaron en los juegos (a 61.8 puntos) en comparación con aquellos que no lo hicieron (a 58.7 puntos). Además, se observó que a medida que avanzaba el nivel académico los conocimientos sobre alimentación nutrición y estilos de vida saludable eran más altos, lo cual puede explicarse -entre otros factores- por la mayor formación académica que se va logrando al transcurrir año tras año.

Aún cuando en la institución intervenida hubo una mejoría significativa en los conocimientos en comparación con la observada en la institución no intervenida, no se alcanzó el puntaje esperado, es decir, un mínimo de 70 puntos. Probablemente, la duración de la intervención con cada uno de los juegos debiera ser mayor; sin embargo, los puntajes alcanzados son satisfactorios si se comparan con otros estudios, en los cuales se obtuvieron resultados similares pero con una gran diferencia en el tiempo de intervención, como en el caso del estudio de Castillo (2) en donde la participación de cada niño fue de por lo menos 8 sesiones por juego, o el juego de Kaledo (6), el cual se desarrolló durante 24 semanas.

Este estudio también buscaba identificar si estos co- nocimientos perduraban en el tiempo. Por ello se aplicó el cuestionario nuevamente tres meses después de realizada la segunda evaluación, encontrándose que dichos conocimientos se mantuvieron $(60.2 \pm 1.1)$ para el grupo de intervención y para el grupo control el promedio fue de (58.8 \pm 1.0$)$. Es necesario enfatizar que a menos que los niños logren un nivel de conocimientos aceptable en alimentación, nutrición y actividad física, no es posible lograr cambios conductuales, propósito final para que un programa de educación en salud sea exitoso. Parece indudable que se requieren evaluaciones a largo plazo para determinar si estos conocimientos se reflejan en cambios de actitud y en mejores condiciones nutricionales y de salud.

Al analizar las diferencias en los conocimientos adquiridos por los estudiantes, mediante la comparación de las puntuaciones antes y después de las intervenciones, indican que los juegos pueden ser un medio educativo de gran valor para lograr un mayor aprendizaje de conceptos de alimentación, nutrición y salud en la población infantil en edad escolar.

Los hallazgos de este estudio están de acuerdo con los reportados por Castillo (2) (2001), Amaro (6) (2006), Munguba (18) (2008) y Wylie (19) (2010), en los que los niños que participaron en los juegos mostraron un cambio positivo en sus niveles de conocimientos sobre alimentación, nutrición y salud, en comparación con los que no participaron. Además, todos coinciden en que se necesitan futuros estudios para determinar si estos conocimientos son perdurables en el tiempo y se ven reflejados en cambios de comportamientos.

Una limitación de este estudio radica en utilizar únicamente un método de medición del desenlace. Así pues, sería de gran utilidad complementar el uso del cuestionario auto administrado con otros métodos, tales como la observación no participante en el aula y la observación de comportamientos de los niños fuera de ella.

Una de las fortalezas del estudio es el tamaño de muestra, con 880 niños escolares distribuidos en dos colegios, muestra muy superior a la reportada por otros estudios $(2,6,18,19)$.

Por medio de estos juegos se pueden orientar a los niños a tener hábitos alimentarios saludables, pero para poder ver resultados reflejados en la modificación de actitudes, prácticas

TABLA 2

Resultados del ajuste del modelo de ecuación de estimación generalizada (GEE) con respuesta continua y estructura de asociación intercambiable.

\begin{tabular}{|c|c|c|c|c|}
\hline Puntaje & Coeficiente & Error estándar & Estadístico de Wald & $\mathrm{p}$ \\
\hline Constante & 23.57 & 8.56 & 2.75 & 0.006 \\
\hline Intervención & -5.10 & 1.40 & -3.64 & 0.000 \\
\hline Momento 2 & 0.57 & 1.20 & 0.47 & 0.638 \\
\hline Momento 3 & 0.63 & 1.24 & 0.51 & 0.612 \\
\hline Intervención* momento 2 & 11.01 & 1.71 & 6.44 & 0.000 \\
\hline Intervención* momento 3 & 9.64 & 1.75 & 5.50 & 0.000 \\
\hline Jornada de tarde & -2.26 & 1.01 & -2.23 & 0.026 \\
\hline Grado 2 & 11.63 & 2.89 & 4.02 & 0.000 \\
\hline Grado 3 & 26.77 & 5.76 & 4.65 & 0.000 \\
\hline Grado 4 & 43.26 & 9.69 & 4.46 & 0.000 \\
\hline Grado 5 & 59.64 & 14.63 & 4.08 & 0.000 \\
\hline Edad & 4.07 & 1.67 & 2.45 & 0.014 \\
\hline Grado *edad & -1.12 & 0.44 & -2.57 & 0.010 \\
\hline
\end{tabular}


y hábitos alimentarios se requiere constancia, por lo que un programa en educación en salud debe estar enmarcado dentro del programa educativo institucional con el fin que exista continuidad en el proceso, involucrando al núcleo familiar y no se convierta en un programa puntual desarrollado en un tiempo específico.

\section{RESUMEN}

Objetivo: Evaluar la efectividad de seis juegos de mesa, en la enseñanza de conceptos de alimentación y nutrición, higiene personal y práctica de actividad física en niños de educación básica. Método: Experimento clínico controlado aleatorizado por conglomerados. Se tomaron dos colegios de Bogotá, D.C. con población escolar (7-12 años) fueron seleccionaron y aleatorizados. En el grupo de intervención se incluyeron 472 niños y en el control 487. Los conocimientos de los niños se evaluaron en tres momentos diferentes. El análisis se realizó utilizando un modelo de ecuaciones de estimación generalizada (GEE) para medidas repetidas. Resultados: El grupo con intervención aumentó su puntaje en el segundo momento, sobre una escala de 0 a 100 pasó de 49.4 a $61.8(p<0.001)$ y se mantuvo en el tiempo. En el grupo control no hubo cambios en el puntaje. Conclusión: Los resultados sugieren que los seis juegos de mesa pueden ser instrumentos efectivos en la enseñanza de conceptos básicos en alimentación, nutrición y salud de niños escolares.

Palabras clave: Efectividad, conceptos, enseñanza, juegos de mesa, nutrición.

\section{BIBLIOGRAFÍA}

1. UNESCO. Perspectivas: revista trimestral de educación comparada., Oficina Internacional de Educación. París; 1993; XXII (3): 501-19.

2. Castillo J, Rodríguez M, Guerrero F. El juego como alternativa para la enseñanza de conceptos básicos de salud. Rev Panam Salud Pública 2001; 9:311-4.

3. Millians D. Simulations and young people: developmental issues and game development. Simulation Gaming 1999; 30(2): 199-226.

4. Corbeil P. Learning from the children: practical and theoretical reflections on playing and learning. Simulation Gaming 1999; 30(2): 163-80.

5. Lennon J. and Coombs D. The utility of a board game for dengue hemorrhagic fever health education. Health Educ. 2007; 107(3): 290-306.
6. Amaro S, Viggiano A, Di Costanzo A, Madeo I, et al. Kalédo, a new educational board-game, gives nutritional rudiments and encourages healthy eating in children: a pilot cluster randomized trial. Eur J Pediatr. 2006; 165:630-5.

7. Plum J, Hertzler A, Brochetti D, Steward D. Game to assess nutrition concepts of preschool children. J Am Diet Assoc. 1998; 98:1168-71.

8. Bandura, A. Social foundation of thought and action: a social cognitive theory. Englewood Cliffs, NJ: PrenticeHall, 1986.

9. Lazcano E, Salazar E, Castrellon P, Angeles A, Hernández $A$, Viramoantes J. Ensayos clínicos aleatorizados: variantes, métodos de aleatorización, análisis, consideraciones éticas y regulación. Salud Pública Mex 2004; 46:559-84.

10. Glaziou P. Sampsize. Sample size and Power Version 0.6. Boston November 9, 2003.

11. Hemming $K$, Girling A, Sitch A, Marsh J and Lilford R. Sample size calculations for cluster randomised controlled trials with a fixed number of clusters. BMC Med Res Methodol. 2011; 11:102.

12. Hayes RJ, Bennett S. Simple size calculation for clusterrandomized trials. Int J Epidemiol. 1999; 28:319-26.

13. Campbell M, Thomson S, Ramsay C, MacLennan G, Grimshaw J. Sample size calculador for cluster randomized trials. Comput Biol Med. 2004; 34:113-25.

14. Lozano JM, Ruiz A. Principios éticos en investigación clínica y epidemiológica. En: Ruiz A, Morillo L. Editores. Epidemiología clínica. Investigación clínica aplicada. Bogotá: Editorial Medica Panamericana, 2004; p 423-34.

15. Rabe S. Everitt B. A Handbook of Statistical Analyses using Stata. Third Edition. Boca Ratón. Florida. Chapman \& Hall. Cap. 10, 2004.

16. Twisk J. Applied Longitudinal Data Analysis for Epidemiology. A Practical Guide. Cambridge University Press 2003; 62-77.

17. Jiang J. Linear and GeneralizedLinear Mixed Models and Their Applications. Springer. 2007.

18. Muguba M, Moreno MT, Bruno CA. The application of an occupational therapy nutrition education programme for children who are obese. OccupTher Int 2008; 15:56-70.

19. Wylie, J, Isasi C, Soroudi N, Soroker E, Sizemore C, PerelsteinG, Bajo J, Diamantis P, Ahmed T, Gandhi R.KidWAVE: Get Healthy Game--promoting a more healthful lifestyle in overweight children. Educ Méx J Nutr 2010; 42:210-2 